old girl who presented to the Emergency Department with non-traumatic right calf pain and swelling.

Case Summary A 4 year old girl presented to Emergency Department (A\&E) with a 3 week history of right calf swelling after her usual, non-traumatic dancing. The swelling had worsened over 48 hours. On examination, her weight was $20 \mathrm{~kg}$, just above the 75 th centile with a BMI of 17.9. She was unable to fully extend her right leg or weight bear due to her calf swelling. Her right calf measured $28.6 \mathrm{~cm}$ compared to the left calf of $26.4 \mathrm{~cm}$. She had been apyrexial with no erythema of the joint. There was no demonstrable joint effusion and no issues with joints above and below. There was no family history of clotting disorder or joint arthropathy.

Ultrasound Scan showed a fluid collection in her right gastrocnemius. MRI confirmed a ruptured Baker's Cyst with inflammatory arthropathy alongside an extensive synovial proliferation throughout the knee joint with large joint effusions and associated soft tissue oedema tracking superiorly and inferiorly along the medial head of gastrocnemius and anteriorly along the tibia. Her was ESR of $26 \mathrm{~mm}$, CRP $<4$ and a normal white cell count. clotting screen and auto-immune panel was negative. investigations revealed bilateral uveitis consistent with a diagnosis of JIA. resolution of joint pain and swelling only occurred after initiation of a short course of oral steroids and steroid injection to the right knee joint.

Clinical Implication This case highlights the atypical presentation of JIA and the clinical manifestations are highly variable in the presence of a ruptured Baker's Cyst. Although, Baker's cysts rare in children. Majority of them are managed conservatively with no other associated comorbidity. To our knowledge, this is the first case of JIA presenting with calf swelling in a toddler

Conclusion We suggest clinicians consider a possible joint inflammatory arthropathy in children presenting with a ruptured Baker's Cyst as early diagnosis and treatment could reduce morbidity associated with this inflammatory arthropathy.

\section{G313 SHOULD RHEUMATIC FEVER AND POST- STREPTOCOCCAL REACTIVE ARTHRITIS BE MANAGED IN THE SAME WAY? A LITERATURE REVIEW}

${ }^{1} Y$ Kulasingham, ${ }^{2} D$ O'Leary. 'Paediatrics, Royal College of Surgeons in Ireland, Dublin, Ireland; ${ }^{2}$ Newman Fellow, UCD Centre for Arthritis Research, Conway Institute, Dublin

10.1136/archdischild-2020-rcpch.271

Aims Although the incidence of rheumatic fever (RF) in the world is declining, the incidence of post-streptococcal reactive arthritis (PSRA) is on the rise. It remains unclear whether both diseases are separate entities or if PSRA is a form of RF. The management of PSRA varies. This review investigates and evaluates the similarities and differences between RF and PRSA, with a specific emphasis on the evidence base for current management guidelines for PSRA in literature.

Methods In this literature review, a focused and methodical search was done on PubMed, Cochrane Library, Google Search, using the words rheumatic fever and post-streptococcal reactive arthritis. Studies which were published between 1999 and 2019 were reviewed with regard to definition, aetiology, epidemiology, clinical presentation, diagnosis and treatment, with the intention of establishing if there is an evidence-based definitive management for post-streptococcal reactive arthritis.

Results In terms of clinical presentation, RF and PSRA present differently. The age at which paediatric patients are affected by both RF and PSRA is similar, although PSRA has a second peak in adulthood. Additionally, the arthritic presentation in $\mathrm{RF}$ is more prolonged (2-3 weeks), whereas in PSRA lasts for 7-10 days. In terms of response to treatment, RF responds better than PSRA, which further indicates the need and importance for defining clear management guidelines for PSRA. There is limited evidence to support antibiotic prophylaxis in PSRA.

Conclusion Current literature supports rheumatic fever and post- streptococcal reactive arthritis as separate entities. However, the management for post-streptococcal reactive arthritis remains unclear. The lack of evidence supporting commonly used management guidelines in PSRA demonstrates the need for further research in this area.

\section{G314(P) A SCOPING REVIEW TO SUPPORT THE DEVELOPMENT OF PGALSPLUS: A MULTI-PROFESSIONAL TOOL AND EDUCATIONAL RESOURCE}

${ }^{1,2}$ VL Mercer, ${ }^{1} \mathrm{~N}$ Smith, ${ }^{3,4} \mathrm{~S}$ Jandial, ${ }^{1,5} \mathrm{HE}$ Foster. ${ }^{1}$ Institute of Cellular Medicine, Newcastle University, Newcastle upon Tyne, UK; ${ }^{2}$ Children's Physiotherapy, South Tyneside and Sunderland NHS FT, South Shields, UK; ${ }^{3}$ Paediatric Rheumatology, Great North Children's Hospital, The Newcastle upon Tyne Hospitals NHS FT, Newcastle upon Tyne, UK; ${ }^{4}$ School of Medical Education, Newcastle University, Newcastle upon Tyne, UK; ${ }^{5}$ Newcastle University Medicine Malaysia (NUMed), Newcastle University, Johor, Malaysia

\subsection{6/archdischild-2020-rcpch.272}

Background Musculoskeletal (MSK) problems in children and young people (CYP) are common. The majority present to healthcare professionals in the community, but it can be challenging to identify those with serious disease. pGALS (paediatric Gait, Arms, Legs and Spine) was developed as a simple MSK clinical assessment to discern abnormal joints, initially within paediatric rheumatology and general paediatrics; pGALS can detect joint and functional problems in CYP with other serious conditions but alone is unlikely to be specific enough to diagnose definitive conditions or signpost referrals to orthopaedics, rheumatology, neurology or general paediatrics. Our aim was to scope the literature about MSK assessments applicable to CYP used in practice, focusing on evidence of validity in the context of diagnosis and assessment of Juvenile Idiopathic Arthritis (JIA), Mucopolysaccharidoses (MPS), Muscular Dystrophy (MD) and Developmental Coordination Disorder (DCD) as exemplar MSK conditions.

Methods Scoping review using a Library search tool (including several databases), Google Scholar, and consulting NICE guidance/pathways. Search terms included dyspraxia, paediatric MSK assessment, screening tools, balance, rheumatology, assessment tools for MD, MPS, JIA. Studies cited within relevant articles were also checked.

Results 32 articles were deemed appropriate, describing specific assessment or screening tools in the context of diagnosis. Within DCD, motor co-ordination batteries aid diagnosis as part of specialist assessment, but are regarded as too lengthy for the purpose of screening; a questionnaire may be a useful first-step diagnostic tool, along with assessment of static balance (found to be significantly worse in children with DCD). In paediatric rheumatology, pGALS is the only validated screening tool to discern normal from abnormal. For 